\title{
Evaluation of Metformin impact on vitamin B12 status
}

\author{
L. Vranken ${ }^{1}$, C. Fontaine ${ }^{1}$, H. Valdes Socin 2 , P. Lukas ${ }^{1}$, C. Le Goff 1, L. Huyghebaert 1, E. Cavalier ${ }^{1}$ \\ ${ }^{1}$ Department of Clinical Chemistry, University of Liège, CHU Sart-Tilman, B-4000 Liège, Belgium. \\ 2 Department of Endocrinology, University of Liège, CHU Sart-Tilman, B-4000 Liège, Belgium \\ Email: laura.vranken@chuliege.be
}

\section{- Introduction}

Metformin is recommended by the American Diabetes Association clinical practice guidelines as the initial glucose-lowering therapy for type 2 diabetes.

Several studies however reported that Metformin treatment on Diabetic patients can be associated with a decrease in serum Vitamin B12 (VB12) concentrations, thus increasing the risk of deficiency, which could have hematological or neurological implications. Similarly to Metformin, gastric acid lowering drugs can reduce circulating Vitamin B12.

Several markers could be used to evaluate Vitamin B12 status, such as total VB12 and holotranscobalamin (active VB12 or HTC) as well as Methylmalonic Acid (MMA) and Homocysteine (HCY) . HTC has been discussed as a more specific and sensitive marker of VB12 deficiency. MMA is also considered to be first line marker for detecting Vitamin B12 deficiency.

\section{- Material and Methods}

Included in the study were 108 consecutive Diabetes patients (58 males and 50 females) under Metformin-treatment for a minimum of 6 months treated in the University hospital in Liege. Metformin dose in these patients ranged from 425 to $3400 \mathrm{mg} /$ day (in 1 to 3 taking per day) and the duration of the treatment ranged from 6 months to 21 years. Mean age of the cohort was 60,9 years (range: 36 to 90 years)

Total VB12, HTC and HCY were performed on Abbott Architect i1000sr instrument. MMA was determined with a in house GC-MS method.

Cut-off

- Serum total VB12 concentrations were classified as deficient $(<200 \mathrm{ng} / \mathrm{L})$, grey-zone (200-300 ng/L) and sufficient (> $300 \mathrm{ng} / \mathrm{L})$

- A cut-off of HTC concentration was set to discriminate deficiency $(<35 \mathrm{pmol} / \mathrm{L})$ and sufficiency (> $35 \mathrm{pmol} / \mathrm{L})$

- Homocysteine (HCY) values $>12 \mu \mathrm{mol} / \mathrm{L}$ were considered deficient and below 12 $\mu \mathrm{mol} / \mathrm{L}$ sufficient.

- For MMA values $>440 \mathrm{nmol} / \mathrm{L}$ were deficient and below $440 \mathrm{nmol} / \mathrm{L}$ sufficient.

Statistical analysis

MedCalc software, version 12.7.7.0 (Oostende, Belgium)

SAS JMP 14 version

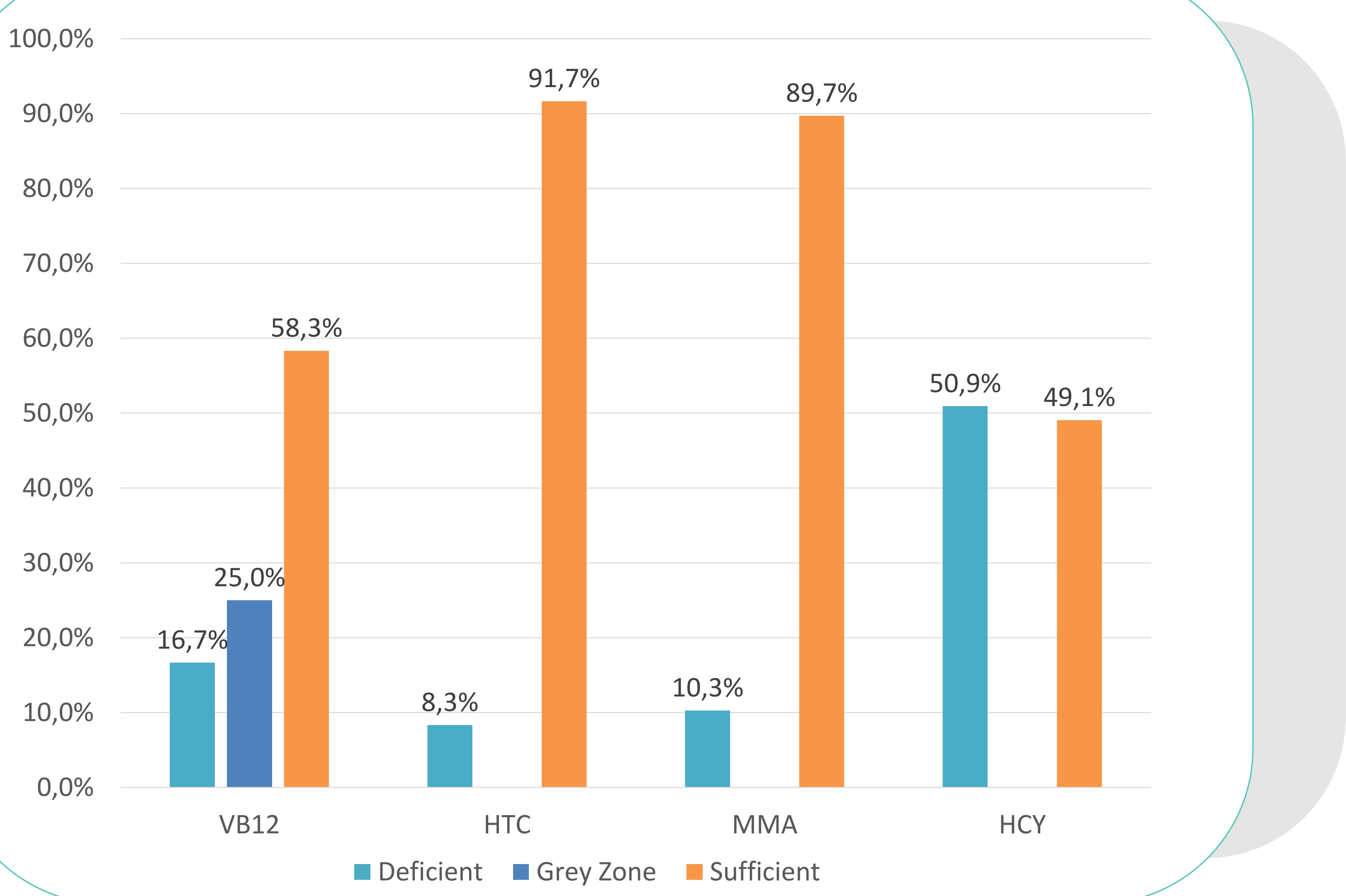

Figure 1 Distribution of deficient patients, grey zone and sufficient patient dependent on VB12, HTC, MMA and HCY

- Aim of the Study

Evaluate the impact of Metformin use in diabetic patients on Vitamin B12 status using different biomarkers associated with Vitamin B12 status.

\begin{tabular}{|c|c|c|c|c|}
\hline & VB12 & MMA & HTC & $\mathrm{HCY}$ \\
\hline \multirow[t]{2}{*}{ VB12 } & & $-0,1571$ & 0,4913 & $-0,0964$ \\
\hline & & 0,1129 & $\leq 0,0001$ & 0,3302 \\
\hline \multirow[t]{2}{*}{ MMA } & $-0,1571$ & & $-0,3771$ & 0,5496 \\
\hline & 0,1129 & & $\leq 0,0001$ & $\leq 0,0001$ \\
\hline \multirow[t]{2}{*}{ HTC } & 0,4913 & $-0,3771$ & & $-0,3379$ \\
\hline & $\leq 0,0001$ & $\leq 0,0001$ & & $\leq 0,0001$ \\
\hline \multirow[t]{2}{*}{ HCY } & $-0,0964$ & 0,5496 & $-0,3379$ & \\
\hline & 0,3302 & $\leq 0,0001$ & $\leq 0,0001$ & \\
\hline
\end{tabular}

Results

Prevalence of Vitamin B12 deficiency in this at-risk population was high. Based on total VB12 status, $16,7 \%$ of the diabetic patients (18 patients) were deficient and $25 \%$ grey-zone deficient (27 patients). For HTC and MMA, 8,3\% of the patients were found to be deficient, and $53 \%$ patients had $\mathrm{HCY}$ values above the threshold for deficiency (Figure 1).

Correlation analysis was conducted among the biomarkers for VB12 status: VB12, HTC $\mathrm{HCY}$ and MMA. Significant correlation for VB12 was only seen for HTC. HTC was however also negatively correlated with $\mathrm{HCY}$ and MMA (Table 1). MMA was negatively correlated with $\mathrm{HTC}$ and positively correlated with $\mathrm{HCY}$.

Logistic Regression model was applied between the dependent and independent variables. Age had significant impact on HTC. With age increase by 1 year, the odds of being MMA deficient was $11,7 \%$ higher, for $\mathrm{HCY}$ it was $6 \%$ higher.

Metformin dose had significant impact on VB12 measurement and the odds of being VB12 non-sufficient was $0,1 \%$ higher than VB12 sufficient if Metformin dose increases by $1 \mathrm{mg}$

For diabetic patients taking gastric acid lowering drugs, the mean HTC was significantly lower than that of patients without Anti Acid. VB12 mean was lower, MMA and HCY means were higher, however there was no statistically significant difference with the patients not taking acid lowering drugs.

The result showed that length of therapy didn't have significant impact on either VB12 or HTC. It had positive significant impact on MMA and HCY, meaning longer length of therapy would result in higher MMA and HCY measurement. Information on possible clinical symptoms in these deficient patients was not available.

\section{- Conclusions}

High prevalence of deficiency was found in this at risk population especially when considering total VB12 results $(16.7 \%$ vs $8.3 \%$ with HTC). More patients were deficient based on total VB12 and HCY results versus HTC or MMA results. This discrepancy may be due to the better specificity of HTC to detect deficiency. This would also confirm the hypothesis that only the inactive part of VB12 is reduced in Metformin-treated patients. VB12 deficiency is a multifactorial phenomenon due to, among others, aging, drugs, malabsorption issues, and malnutrition. Careful clinical practice would be to evaluate and follow-up VB12 status before and during Metformin treatment. American Diabetes Association guidelines recommend regular testing in treated patients, especially if peripheral neuropathy is present. HTC seems to be able to better determine Vitamin B12 status

\section{- References}

American Diabetes Association. Approaches to glycemic treatment. Diabetes Care 2015; 38 (suppl): S41-48.

- Rena G, D. Hardie DG, Pearson ER, The mechanisms of action of metformin, Diabetologia (2017) 60:1577-1585

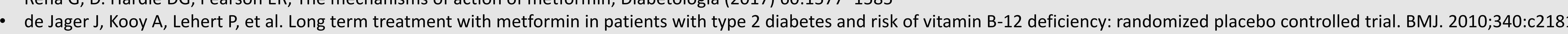

